\title{
Novel Ideas for Fast Muscle Action Potential Simulations Using the Line Source Model
}

\author{
Björn Hammarberg (Hansson)*, Member, IEEE, and Erik Stålberg
}

\begin{abstract}
Using a signal processing approach, we analyze the line source model for muscle action potential (AP) modeling. We show that the original model presents a tradeoff between violating the Nyquist criterion on one hand and using a discretization frequency that is unnecessarily high with respect to the bandwidth of the generated AP on the other. Here, we present an improved line source model that, compared to the original, allows a lower discretization frequency while retaining the accuracy by simply introducing a continuous-time anti-aliasing filter. Moreover, a transfer function form of the transmembrane current is presented that promote the use of sophisticated signal processing methods on these type of signals. Both continuous-time and discrete-time models are presented. We also address and analyze the implications of the finite length of the muscle fibers. Including this in the model is straightforward, owing to the convolutional form of the line source model, and is manifested by a simple transformation of the associated weighting function. AP modeling is discussed for the three different electrode models: the concentric needle electrode, the single fiber electrode, and the macro electrode. The presented model is suitable for modeling large motor units, where both accuracy and computational efficiency are important factors. To simplify the selection of the discretization interval, we derive what we call the cumulative cutoff frequency that provides an estimate of the required Nyquist frequency.
\end{abstract}

Index Terms-Action potential, aliasing, electromyography (EMG), line source model, modeling, simulation.

\section{INTRODUCTION}

$\mathbf{R}$ ELIABILITY and efficiency when diagnosing muscle and nerve disorders using electromyography (EMG) is essential. To improve both aspects, the relationship between the physiological conditions and the electric signal of the muscle upon activation must be accurately known. A mathematical model has several advantages in this respect. Besides being a compact description of the present knowledge, it may also be used for simulations to obtain new insights. Also, and perhaps more intriguing, a mathematical description makes it conceivable to use signal processing methods for "digital dissection," where the physiological parameters are presented to the clinician.

To measure the generated electrical activity, the EMG, when the muscle is activated, different recording electrodes may be used. The three most common ones, the concentric needle $(\mathrm{CN})$

\footnotetext{
Manuscript received November 16, 2001; revised March 10, 2003. This work was supported by the Swedish Medical Research Council (ES, 135). Asterisk indicates corresponding author.

*B. Hammarberg is with Signals and Systems, Uppsala University, P.O. Box 28, Uppsala SE-75120, Sweden, and also with the Department of Clinical Neurophysiology, University Hospital, Uppsala SE-75120, Sweden (e-mail: Bjorn.Hammarberg@ signal.uu.se).

E. Stålberg is with the Department of Clinical Neurophysiology, University Hospital, Uppsala SE-75120, Sweden.

Digital Object Identifier 10.1109/TBME.2004.834292
}

electrode [1], [2], the single fiber (SF) electrode [3], [4], and the macro electrode [5], are considered below.

The knowledge about the electrical events in the muscle fiber membrane and the volume conduction characteristics of muscle tissue has made it possible to simulate the action potentials (APs) as if they were recorded with a particular electrode. Without simplification, however, volume conduction theory [6]-[8] leads to complex and time-consuming calculations.

Various approaches to this accuracy/complexity tradeoff have been presented [9]-[16]. By lumping the transmembrane current to the center of the muscle fiber, the line source model is obtained which describes the AP as a convolution of a weighting function and the transmembrane current [13].

Aliasing may arise if the Nyquist criterion is violated when the line source model is discretized. This crucial aspect has not been properly addressed in the literature. In this paper, we identify the sources of aliasing and present a design variable that simplifies the selection of a proper discretization frequency.

Often, fast simulations are needed. We meet this requirement by introducing an anti-aliasing filter that lowers the required dicretization frequency. This makes it possible to improve the performance of the simulations by either decreasing the calculation time, increasing the accuracy, or attaining both objectives.

Recently, the importance of the finite length of the muscle fibers has been studied [14]-[19]. Assuming a finite muscle fiber length in a line source model is straightforward but has to be done properly to avoid the introduction of artifacts in the model output. To achieve this, either the discontinuities have been handled separately from the convolution [19] or the transmembrane current has been replaced by the dipolar source strength [15]. We show that it is possible to account for the finite fiber length by a simple transformation of the weighting function instead.

The focus of this paper is on modeling of single muscle fiber APs, with simulation of entire motor units (MUs) and different measuring electrodes in mind. The efficiency of the calculations is therefore a very important aspect in which the anti-aliasing filter and the weighting function transformation play a central role.

In addition to simulation, accurate models may be used in filter design such as deconvolution of the EMG signal. Describing the model in a transfer function form, as presented in this paper, is beneficial in this perspective. The ultimate goal would then be the situation where the EMG signal, obtained with the $\mathrm{CN}$ electrode, is decomposed into single muscle fiber AP components, using model-based deconvolution.

The paper is organized as follows: Section II gives the preliminaries of the line source model for finite length fibers. In 


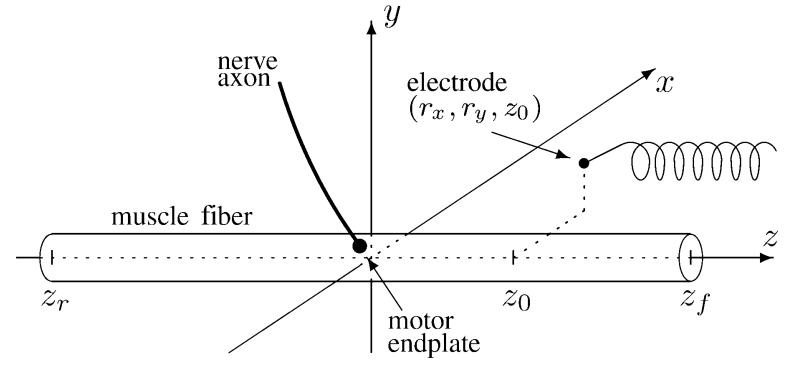

Fig. 1. The nerve impulse is conducted along the peripheral nerve axon and triggers the muscle fiber at the motor endplate. This results in a depolarization of the muscle fiber membrane that, in turn, causes a transmembrane current to appear and spread toward the two fiber endings where it vanishes. This distribution in both time and space of the transmembrane current is then measured by the electrode.

Section III, the continuous-time line source model is presented. In Section IV, the model is discretized into a discrete-time model. The cause of the aliasing errors is presented and an anti-aliasing filter is introduced. In Section V, the three common electrode types are presented. Simulation results are presented in Section VI, followed by a concluding discussion in Section VII.

\section{MODEl PRELIMINARIES}

The muscle fiber receives a nerve impulse from the axon and becomes depolarized (see Fig. 1). The depolarization generates a transmembrane current that originates at the motor end plate and propagates along the fiber toward the two tendons.

The potential measured by a particular electrode is then the spatially weighted summation of the time-dependent transmembrane current distribution along the fiber (see Fig. 2). Denoting the time-space distribution of the transmembrane current with $\xi(t, z)$ and the electrode-dependent weighting function (spatial) with $\bar{w}(z)$, the recorded AP, denoted $\phi_{c}(t)$, is given by ${ }^{1}$

$$
\phi_{c}(t)=\int_{-\infty}^{\infty} \bar{w}(z) \xi(t, z) d z
$$

Assuming the fiber properties are constant along the fiber, the transmembrane current distribution $\xi(t, z)$ may be described as a propagation of a source function $i_{c}(\cdot)$. Fig. 2 shows $\xi(t, z)$ graphically where two sources are generated at the motor end plate and move in opposite directions with a constant velocity $c$. The transmembrane current that moves in the forward direction (increasing $z$ ) is denoted $i_{f}(t, z)$ and the transmembrane current that moves in the reverse direction (decreasing $z$ ) is denoted $i_{r}(t, z)$. Both currents vanish at the fiber endings where the depolarization regeneration ceases. Obviously, these two functions may be formulated as

$$
\begin{aligned}
& i_{f}(t, z)= \begin{cases}i_{c}\left(t-\frac{z}{c}\right), & 0 \leq z \leq z_{f} \\
0, & \text { otherwise }\end{cases} \\
& i_{r}(t, z)= \begin{cases}i_{c}\left(t+\frac{z}{c}\right), & z_{r} \leq z \leq 0 \\
0, & \text { otherwise }\end{cases}
\end{aligned}
$$

where both $i_{f}(t, 0)$ and $i_{r}(t, 0)$ are equal to $i_{c}(t)$.

\footnotetext{
${ }^{1}$ Throughout this paper, the index ${ }_{c}$ is used to distinguish continuous-time from discrete-time entities.
}

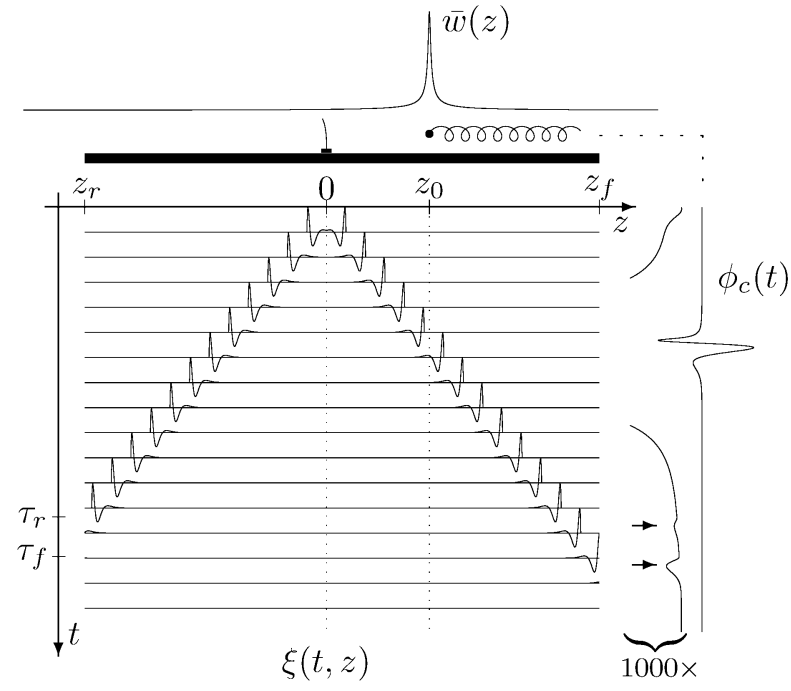

Fig. 2. The generation of an AP, denoted $\phi_{c}(t)$, using the line source model is illustrated in a two-dimensional spatio-temporal view. The spatial dimension is along the axial direction of the muscle fiber and is drawn from left to right in the figure. The temporal dimension is drawn from top to bottom where time increases downwards. At $t=0$, a depolarization is initiated at the motor end plate $(z=0)$ causing two transmembrane currents to move toward the fiber endings where they vanish at $t=\tau_{f}\left(z=z_{f}\right)$ and $t=\tau_{r}\left(z=z_{r}\right)$, respectively. The electrode is positioned at $z=z_{0}$ and records the resulting AP, i.e., the time-space transmembrane current distribution, $\xi(t, z)$, weighted by the electrode characteristic spatial weighting function, $\bar{w}(z)$. Next to the resulting AP is an enlargement of it with the central part excluded. The end effects, or terminal waves, originating from the two muscle-tendon junctions are indicated by arrows. Note the absence of a corresponding motor end plate effect at $t=0$.

To obtain the final expression of $\phi_{c}(t)$, any possible end effects taking place in the discontinuities at the motor end plate and at the two fiber endings, respectively, are considered. Therefore, the time-space distribution of the transmembrane current $\xi(t, z)$ is defined as

$$
\begin{aligned}
\xi(t, z) \triangleq i_{f}(t, z)+ & i_{r}(t, z)+\psi_{0}(t) \delta(z) \\
& +\psi_{1}(t) \delta\left(z-z_{f}\right)+\psi_{2}(t) \delta\left(z-z_{r}\right)
\end{aligned}
$$

where the $\psi_{i}(t)$ 's, $i=0,1,2$, account for the discontinuities.

By using (4) and the substitution $z=c t$ to expand (1) (see Appendix A), the measured potential $\phi_{c}(t)$ becomes a convolution between the transmembrane current $i_{c}(t)$ and a temporal weighting function $w_{c}(t)$ according to

$$
\phi_{c}(t)=i_{c}(t) * w_{c}(t) \triangleq \int_{0}^{t} w_{c}(\tau) i_{c}(t-\tau) d \tau
$$

\section{Continuous-Time Model}

As shown above, the potential measured by a particular electrode may be modeled as a convolution of a transmembrane current and an electrode-dependent weighting function. The measured $\mathrm{AP} \phi_{c}(t)$ from one fiber may thus be modeled by

$$
\phi_{c}(t)=W_{c}(p) I_{c}(p) x_{c}(t)
$$

or, in the frequency domain

$$
\Phi_{c}(s)=W_{c}(s) I_{c}(s) X_{c}(s)
$$




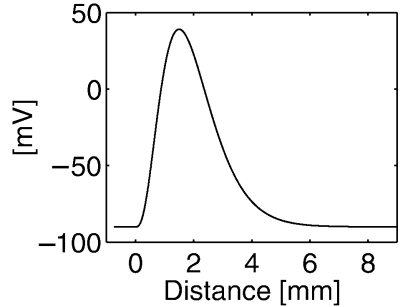

(a)

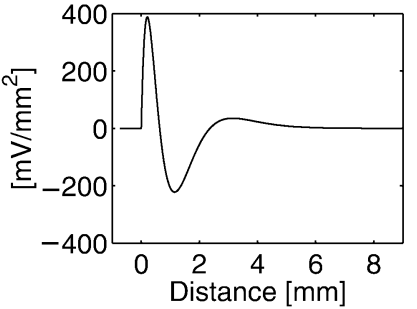

(b)
Fig. 3. Instantaneous spatial distribution along the fiber of (a) the intracellular potential and (b) its second derivative.

where $p$ is the derivative operator $\left(p x_{c}(t)=(d / d t) x_{c}(t)\right)$. The impulse train delivered by the axon is described by $x_{c}(t)$ and $X_{c}(s)$ in the time domain and the frequency domain, respectively. The electrode-dependent weighting function and the transmembrane current are modeled in the frequency domain by $W_{c}(\cdot)$, and $I_{c}(\cdot)$, respectively.

To simplify the notation and the calculations below, we assume that $x_{c}(t)$ contains only one pulse at $t=0$, i.e., $x_{c}(t)=$ $\delta(t)$, where $\delta(t)$ is the Dirac function. Extending this to the general case is straightforward.

\section{A. Temporal Transmembrane Current}

Assuming linearity, the relation between the source function $i_{c}(t)$ and the intracellular action potential (IAP) $\psi_{c}(t)$ may be modeled as

$$
i_{c}(t)=\frac{\sigma_{i} \pi d^{2}}{4} G(p) \psi_{c}(t)
$$

where $G(p)$ is a linear function in the operator $p$. In this paper, we follow the Rosenfalck approach with $G(p)=\left(1 / c^{2}\right) p^{2}$, which means

$$
G(p) \psi_{c}(t)=\frac{1}{c^{2}} p^{2} \psi_{c}(t)=\frac{1}{c^{2}} \frac{d^{2}}{d t^{2}} \psi_{c}(t) .
$$

Other, more complex and more realistic, filters may of course be designed. In [20], the authors concluded that the relation between the IAP and the transmembrane current is more complex than a simple double differentiation.

For many applications, however, the Rosenfalck approach is sufficiently accurate. Following [12], the IAP used for the simulations in this paper is given by (see Fig. 3)

$$
\begin{aligned}
\psi_{c}(t) & \triangleq \begin{cases}768 c^{3} t^{3} e^{-2 c t}-90, & t \geq 0 \\
0, & \text { otherwise } \quad[\mathrm{mV}]\end{cases} \\
\Psi_{c}(s) & =\mathcal{L}\left\{\psi_{c}(t)\right\}=768 \frac{6 c^{3}}{(s+2 c)^{4}}
\end{aligned}
$$

in the time domain and the frequency domain, respectively.

Combining (11) and (8), we obtain the sought transmembrane current in the frequency domain $I_{c}(s)$ as

$$
I_{c}(s)=\frac{\sigma_{i} \pi d^{2}}{4} \frac{s^{2}}{c^{2}} \Psi_{c}(s)=768 \frac{\sigma_{i} \pi d^{2}}{4} \frac{6 c s^{2}}{(s+2 c)^{4}} .
$$

As is generally accepted, we assume a linear relationship between conduction velocity and fiber diameter, using a relation proposed in [12]

$$
c \triangleq 3.7+50\left(d-55 \cdot 10^{-3}\right)\left[\frac{\mathrm{m}}{\mathrm{s}}\right] .
$$

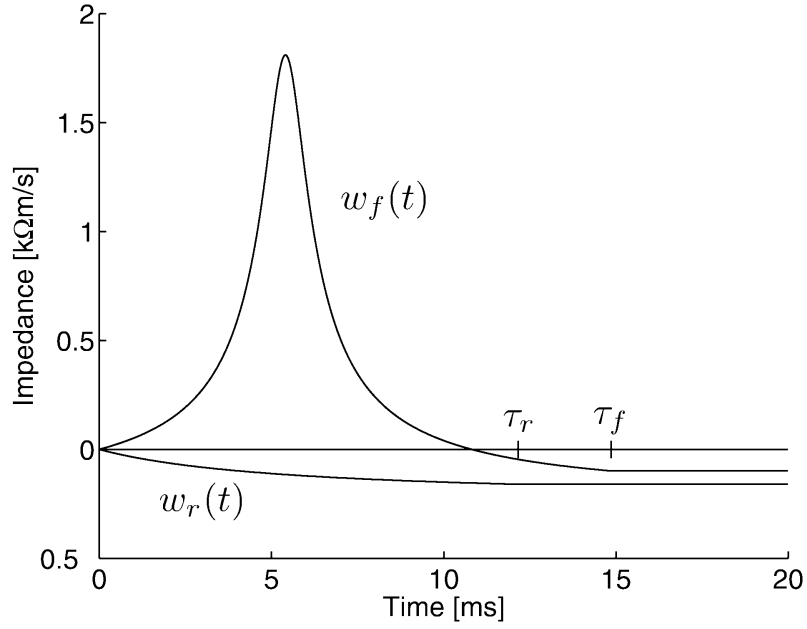

Fig. 4. Example of a temporal weighting function in the forward $w_{f}(t)$ and the reverse $w_{r}(t)$ directions for a point electrode at a radial distance of $1 \mathrm{~mm}$. The conduction velocity is set to $3.7 \mathrm{~m} / \mathrm{s}$ and the length of the muscle fiber is $100 \mathrm{~mm}$. Due to the noncentered end plate position, the arrival times $\left(\tau_{f}\right.$ and $\left.\tau_{r}\right)$ at the fiber tendon differ. Note that each weighting function is constant for $t>\tau_{f}$, and $t>\tau_{r}$, respectively.

\section{B. Temporal Weighting Function}

The temporal weighting function $w_{c}(t)$ corresponding to $W_{c}(s)$ in (7) is derived from the spatial weighting function $\bar{w}(z)$, here assumed known. The function $\bar{w}(z)$ is electrode dependent and describes the potential produced by a unit current source located on a straight line at a certain radial distance (see Section V).

Through the derivation of $w_{c}(t)$, one actually obtains one weighting function in each direction, $w_{f}(t)$ and $w_{r}(t)$, because $i_{f}(t, z)$ is affected by $\bar{w}(z)$ for $z \geq 0$ only and $i_{r}(t, z)$ is affected by $\bar{w}(z)$ for $z \leq 0$ only (see Appendix A and Fig. 4). For interpretational reasons, this division is kept throughout this paper. The two functions are related to $w_{c}(t)$ as

$$
\begin{aligned}
& w_{c}(t) \triangleq w_{f}(t)+w_{r}(t) \\
& w_{f}(t)= \begin{cases}c(\bar{w}(c t)-\bar{w}(0)), & 0 \leq t<\tau_{f} \\
c\left(\bar{w}\left(z_{f}\right)-\bar{w}(0)\right), & t \geq \tau_{f} \\
0, & \text { otherwise }\end{cases} \\
& w_{r}(t)= \begin{cases}c(\bar{w}(-c t)-\bar{w}(0)), & 0 \leq t<\tau_{r} \\
c\left(\bar{w}\left(z_{r}\right)-\bar{w}(0)\right), & t \geq \tau_{r} \\
0, & \text { otherwise }\end{cases} \\
& \tau_{f}=\frac{z_{f}}{c} ; \quad \tau_{r}=\frac{-z_{r}}{c} .
\end{aligned}
$$

Owing to the division of the weighting function, an informal interpretation of the transformation of a spatial weighting function $\bar{w}(z)$ to a temporal weighting function $w_{c}(t)$ is possible as follows (see Figs. 2 and 4). The spatial weighting function $\bar{w}(z)$ is translated vertically so that it is zero at the motor end plate $(\bar{w}(0)+$ const $=0)$. Then, the value at each fiber ending is extrapolated to infinity $\left(\bar{w}(z)=\bar{w}\left(z_{f}\right) \forall z \geq z_{f}, \bar{w}(z)=\right.$ $\left.\bar{w}\left(z_{r}\right) \forall z \leq z_{r}\right)$. The part to the left of the motor end plate $(\{\bar{w}(z): z<0\})$ is "folded" to the right and added to the part to the right of the motor end plate $(\{\bar{w}(z): z>0\})$. The transformation is then completed by the substitution $z=c t$.

This concludes the derivation of the continuous-time representation of a measured AP. This representation is most useful 
for understanding the underlying relations of the AP generation. For simulations and signal processing, it is convenient to use the discrete-time representation derived next.

\section{DisCRETE-TIME MODEL}

In this section, a discrete-time representation of the line source model is derived through impulse-invariant discretization (sampling) of the continuous-time model (6). This approach is straightforward and well known [19], [21]. Using a discretization interval $T$, the discrete-time model may be written as

$$
\phi(n)=W(q) I(q) x(n)
$$

where $\phi(n)$ is the discretized potential $\left(\approx \phi_{c}(n T)\right), q$ is the forward shift operator $(q x(n)=x(n+1)), W(q)$ is the electrodedependent weighting function, $I(q)$ models the transmembrane current, and $x(n)$ is the impulse train delivered by the axon.

To avoid aliasing when discretizing the continuous-time model functions, the Nyquist criterion has to be fulfilled for these functions. The aliasing issues, however, have not been addressed properly in the literature. When addressed, it has been the bandwidth of the resulting $A P$ that has been considered in the Nyquist criterion. This is not correct because, in the discrete-time line source model, the transmembrane current and the weighting function are discretized prior to the convolution that generates the resulting AP. In essence, the discrete-time AP should not be regarded as sampled since it is generated in discrete time directly. ${ }^{2}$ As shown in Section VI-B, the transmembrane current has a significant high-frequency content that cannot be seen in the resulting AP.

The most important and noticeable consequence of the aliasing is the appearance of a static error in the resulting AP. This static error is the summed contribution from all spectral components at multiples of the sampling frequency. When modeling entire MUs with as many as 500 fibers or even more, it is very important that the static errors are vanishingly small. Even small magnitude errors can, in such a case, be amplified to a dominant source of error. This in combination with the demand for fast simulations causes a tradeoff between accuracy and low discretization frequency.

By properly addressing these issues, a discrete-time model is possible to derive that is optimized for accurate and computationally efficient computer based modeling of muscle fiber APs. Our approach, obvious when sampling real signals, is to use anti-aliasing prefiltering within the model, which allows significantly lower discretization frequencies. To understand this, assume, for example, that we have an AP with a 5-kHz bandwidth In general, this corresponds to a transmembrane current with a bandwidth of $20 \mathrm{kHz}$ and a weighting function with a bandwidth of $5 \mathrm{kHz}$. Hence, despite the bandwidth of the AP being $5 \mathrm{kHz}$ in this example, aliasing-free discretization requires a discretization frequency of $40 \mathrm{kHz}$. Using an anti-aliasing filter to remove the excess bandwidth of the transmembrane current, the discretization frequency may be lowered by as much as a factor

\footnotetext{
${ }^{2}$ Hence, the discrete-time AP, $[\phi](\mathrm{n})$, is only an approximation to the continuous-time AP, $\left[\phi_{c}\right](\mathrm{n})$, where the accuracy is directly related to the amount of aliasing errors.
}

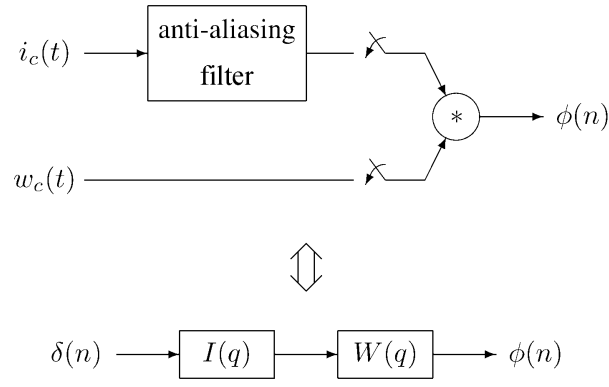

Fig. 5. Block diagram of the discretized model.

four to $10 \mathrm{kHz}$. This indicates that the anti-aliasing filter improves the efficiency while retaining the accuracy.

There are principally three ways of implementing the antialiasing filter.

1) Analytically (time domain): By applying an anti-aliasing filter to the continuous-time model and find the closed form of the filtered replica.

2) Seminumerically (frequency domain): By implementing the convolution using the fast Fourier transform (FFT) algorithm, it is sufficient to know a finite number of discrete points on the unit circle of, e.g., the transmembrane current $I(\zeta)$.

3) Numerically (time domain): By first discretizing the model using a high sampling frequency and low-pass filtering it numerically, the resulting model may be discretized at the desired rate. To account for different, e.g., conduction velocity, the discretized model needs to be interpolated or rediscretized.

The choice that is most beneficial is determined by the particular application. In this paper, we use the analytical approach because it is best suited for visualization. The so obtained discrete-time model is shown in Fig. 5. As the anti-aliasing filter, we use a Bessel filter of the second order (see Appendix B).

\section{A. Transmembrane Current Model}

After prefiltering the continuous-time transmembrane current using an anti-aliasing filter, the actual discretization of the resulting continuous-time model is straightforward. We derive the discrete-time transmembrane current per unit length of fiber $i(n)$ through impulse-invariant discretization of the prefiltered analog using a discretization interval $T$ as described by

$$
i(n)=i_{c}^{(H)}(c n T)\left[\frac{\mu \mathrm{A}}{\mathrm{mm}}\right]
$$

where $i_{c}^{(H)}(\cdot)$ is given in Appendix B.

\section{B. Weighting Function Model}

As mentioned above, the weighting function has the lowest bandwidth (similar to the bandwidth of $\phi(n)$ ) of the two generating model functions. This means that if the discretization frequency is sufficiently high to accomodate the bandwidth of the continuous-time AP, the aliasing errors in the discretization of the weighting function is small. Since this is the normal case, no anti-aliasing filter for the weighting function is presented. The discrete-time weighting function is then directly derived 
TABLE I

ELECTRODE SPECIFICATIONS

\begin{tabular}{lccr} 
Description & \multicolumn{2}{c}{ Parameter } & Value \\
\hline \hline Needle radius & $R_{n}$ & {$[\mathrm{~mm}]$} & 0.225 \\
\hline Needle inner radius & $R_{e}$ & {$[\mathrm{~mm}]$} & 0.150 \\
\hline CN core radius & $R_{C}$ & {$[\mathrm{~mm}]$} & 0.075 \\
\hline CN recording angle & $\theta_{C N}$ & {$[\mathrm{rad}]$} & $\frac{\pi}{12}$ \\
\hline CN cannula length & $L_{C N}$ & {$[\mathrm{~mm}]$} & 20 \\
\hline Macro cannula length & $L_{M}$ & {$[\mathrm{~mm}]$} & 15 \\
\hline SF core radius & $R_{S F}$ & {$[\mathrm{~mm}]$} & 0.0125 \\
\hline SF recording position & $x_{S F}$ & {$[\mathrm{~mm}]$} & $\frac{1}{2} L_{M}$ \\
\hline
\end{tabular}

through impulse-invariant discretization of the continuous-time model $w_{c}(t)$ in (14) using a discretization interval $T$ as described by

$$
w(n)=w_{c}(n T)\left[\frac{\mathrm{k} \Omega \cdot \mathrm{m}}{\mathrm{s}}\right]
$$

\section{Electrode-Characteristic Weighting Functions}

In the simulations (see Section VI), we present the performance of the line source model. Here, we recapitulate the weighting functions assumed known in Sections III and IV.

The physical dimensions of standard SF, CN, and macro electrodes are listed in Table I.

\section{A. Impedance of a Point Electrode}

In an infinite medium with cylindrical anisotropy, a current source $\iota_{g}$ located at the origin produces a potential $\phi(x, y, z)$ in a point electrode positioned at $(x, y, z)$ (see Fig. 1) by [13], [21]

$$
\begin{aligned}
& \phi(x, y, z)=Z(x, y, z) \iota_{g} \\
& Z(x, y, z)=\frac{1}{4 \pi \sigma_{r}} \frac{1}{\sqrt{K\left(x^{2}+y^{2}\right)+z^{2}}}\left[\frac{\mathrm{k} \Omega \cdot \mathrm{m}}{\mathrm{s}}\right]
\end{aligned}
$$

where $Z(x, y, z)$ is the impedance of the point electrode, $\sigma_{r}$ is the radial conductivity, $\sigma_{z}$ is the axial conductivity, and $K \triangleq$ $\left(\sigma_{z} / \sigma_{r}\right)$ is the anisotropy ratio.

\section{B. Electrode Weighting Functions}

Assuming that a muscle fiber may be approximated by a straight and cylindrical entity, and that the potential produced in a particular electrode is the average of the potential present on the recording surface, the following three models were derived: the macro electrode, the SF electrode, and the CN electrode. The latter two are bipolar and use the cannula as reference. More accurate models exist [22], [23], but their complexity and computational demand often outweigh their improvement in accuracy.

The three weighting functions below are derived using the electrode specifications listed in Table I and assuming the electrode is held perpendicular to the fiber.

1) Macro Electrode and Cannula Reference: Approximating the cylindrical recording surface with a thin wire of length $L$, the weighting functions of the macro electrode $\left(L=L_{M}\right)$ and the $\mathrm{CN}$ cannula $\left(L=L_{\mathrm{CN}}\right)$ are the average

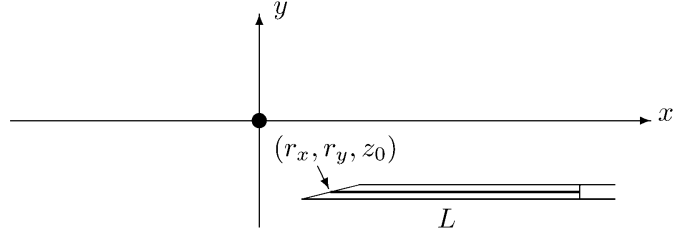

Fig. 6. The macro electrode and the cannula of the $\mathrm{CN}$ electrode are approximated by a wire (thick line) of length $L=L_{M}$ and $L=L_{\mathrm{CN}}$, respectively. The left end of the wire is at the position $\left(r_{x}, r_{y}\right)$. The muscle fiber is perpendicular to the needle and located at the origin.

over its length [9], [24] (see Fig. 6). For the macro electrode, we thus obtain

$$
\begin{aligned}
\bar{w}_{M}(z) \triangleq & \frac{1}{L_{M}} \int_{L_{M}} Z d L \\
= & \frac{1}{4 \pi \sigma_{r} \sqrt{K} L_{M}} \\
& \ln \frac{\sqrt{\left(r_{x}+L_{M}\right)^{2}+B}+r_{x}+L_{M}}{\sqrt{r_{x}^{2}+B}+r_{x}}\left[\frac{\mathrm{k} \Omega \cdot \mathrm{m}}{\mathrm{s}}\right]
\end{aligned}
$$

with $B=r_{y}^{2}+(1 / K)\left(z-z_{0}\right)^{2}$.

The weighting function $\bar{w}_{\mathrm{CN} c}(z)$ of the $\mathrm{CN}$ electrode cannula is calculated analogously using

$$
\bar{w}_{\mathrm{CN} c}(z) \triangleq \frac{1}{L_{\mathrm{CN}}} \int_{L_{\mathrm{CN}}} Z d L .
$$

2) SF Electrode: The active surface of the SF electrode is located in a side port $7.5 \mathrm{~mm}$ from the tip of the electrode. Neglecting the so-called wall effect [12], the weighting function of the active surface is approximated by a single point due to its dimunitive dimension as described by

$$
\begin{aligned}
\bar{w}_{\mathrm{SF} a}(z) & \triangleq Z\left(-\left(r_{x}+x_{\mathrm{SF}}\right),-\left(r_{y}+R_{n}\right), z-z_{0}\right) \\
& =\frac{1}{4 \pi \sigma_{r} \sqrt{K r^{2}+\left(z-z_{0}\right)^{2}}}\left[\frac{\mathrm{k} \Omega \cdot \mathrm{m}}{\mathrm{s}}\right]
\end{aligned}
$$

where $r=\sqrt{\left(r_{x}+x_{\mathrm{SF}}\right)^{2}+\left(r_{y}+R_{\mathrm{SF}}\right)^{2}}$ is the radial distance translated by the off-tip position of the SF core (see Table I).

The final weighting function of the bipolar SF electrode is

$$
\bar{w}_{\mathrm{SF}}(z) \triangleq \bar{w}_{\mathrm{SF} a}(z)-\bar{w}_{M}(z) .
$$

3) CN Electrode: Approximating the surface of the central core of the $\mathrm{CN}$ electrode by $2 N_{c}+1$ parallell wires (see Fig. 7), the weighting function $\bar{w}_{\mathrm{CNa}}(z)$ of the active surface of the $\mathrm{CN}$ electrode is [21]

$$
\begin{aligned}
\bar{w}_{\mathrm{CN} a}(z) & \triangleq \frac{1}{S} \iint_{S} Z d S \\
\approx & \frac{1}{2 N_{c}+1} \sum_{k=-N_{c}}^{N_{c}} \frac{1}{8 \pi \sigma_{r} \sqrt{K} \Delta u_{k}} \\
& \times \ln \frac{\sqrt{\left(r_{u}+\Delta u_{k}\right)^{2}+B_{k}}+r_{u}+\Delta u_{k}}{\sqrt{\left(r_{u}-\Delta u_{k}\right)^{2}+B_{k}}+r_{u}-\Delta u_{k}}\left[\frac{\mathrm{k} \Omega \cdot \mathrm{m}}{\mathrm{s}}\right] \\
B_{k} & =r_{v}^{2}+\frac{1}{K}\left(z-\left(\Delta z_{k}+z_{0}\right)\right)^{2} \\
\Delta z_{k} & =R_{c} \frac{k}{N_{c}+1}, \quad k=-N_{c}, \ldots, N_{c}
\end{aligned}
$$




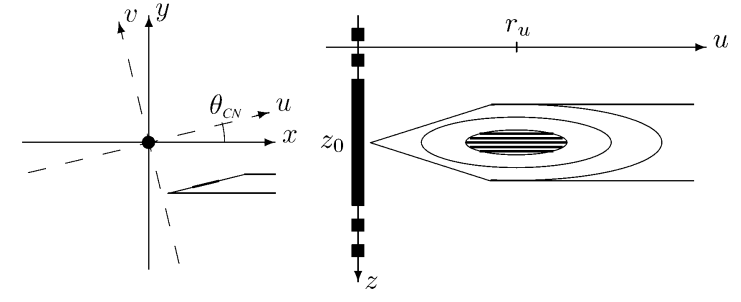

Fig. 7. The core of the $\mathrm{CN}$ electrode is approximated by five parallel wires (thick lines). To simplify the calculations, an alternate coordinate system $(u, v)$ is introduced. It is rotated by the angle $\theta_{C N}$ and the $u$-axis is parallel with the recording surface on the beveled tip of the $\mathrm{CN}$ electrode.

TABLE II SIMULATION PARAMETERS

\begin{tabular}{llcr} 
Description & \multicolumn{2}{l}{ Parameter } & Value \\
\hline \hline Fiber diameter & $d$ & {$[\mathrm{~mm}]$} & $55 \cdot 10^{-3}$ \\
\hline Conduction velocity & $c$ & {$[\mathrm{~m} / \mathrm{s}]$} & 3.7 \\
\hline Radial distance & $r$ & {$[\mathrm{~mm}]$} & 0.1 \\
\hline Axial distance & $z_{0}$ & {$[\mathrm{~mm}]$} & 20 \\
\hline Filter cut-off frequency & $f_{0}$ & {$[\mathrm{kHz}]$} & 5.8 \\
\hline Intracellular conductivity & $\sigma_{i}$ & {$[\mathrm{~S} / \mathrm{m}]$} & 1.01 \\
\hline Axial conductivity & $\sigma_{z}$ & {$[\mathrm{~S} / \mathrm{m}]$} & 0.33 \\
\hline Radial conductivity & $\sigma_{r}$ & {$[\mathrm{~S} / \mathrm{m}]$} & 0.063 \\
\hline CN core approximation size & $N_{c}$ & & 2 \\
\hline
\end{tabular}

$$
\begin{aligned}
\Delta u_{k} & =\frac{R_{c}}{\sin \left(\theta_{\mathrm{CN}}\right)} \cos \left(\arcsin \left(\frac{k}{N_{c}+1}\right)\right) \\
r_{u} & =r_{x} \cos \theta_{\mathrm{CN}}+r_{y} \sin \theta_{\mathrm{CN}} \\
r_{v} & =-r_{x} \sin \theta_{\mathrm{CN}}+r_{y} \cos \theta_{\mathrm{CN}}
\end{aligned}
$$

where $\theta_{C N}$ is the angle of the beveled tip of the $\mathrm{CN}$ electrode.

The weighting function of the bipolar $\mathrm{CN}$ electrode is [25]

$$
\bar{w}_{\mathrm{CN}}(z) \triangleq \bar{w}_{\mathrm{CN} a}(z)-\bar{w}_{\mathrm{CN} c}(z) \text {. }
$$

\section{Simulation Results}

In this section, the characteristics of the model are visualized through simulations. We present some sample APs, the frequency distribution, and bandwidth of the model components. Moreover, the aliasing effects are illustrated. In all simulations, unless stated differently, the parameter settings used are found in Table II.

\section{A. AP Simulation}

The most time-consuming part of the line source model evaluation is the convolution of the transmembrane current and the electrode characteristic weighting function. For fast evaluation of the convolution, it is calculated in the frequency domain via the FFT algorithm [12]. When doing that, both the weighting function and the transmembrane current must be discretized using the same discretizing frequency and must be padded with zeroes to their double length. The latter operation is to avoid circular convolution. After taking the inverse FFT, the resulting potential is truncated to the length of the original vectors.

In Fig. 8, three APs calculated using this technique are shown. Each is a sample of an AP recorded by the SF electrode (SFAP),

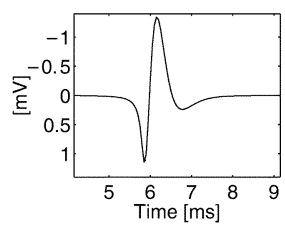

(a)

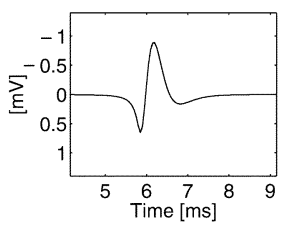

(b)

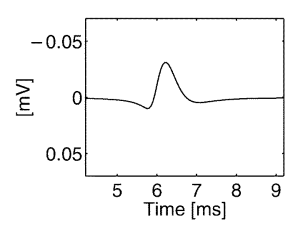

(c)
Fig. 8. Simulations of an AP as recorded with (a) the SF electrode (SFAP), (b) the CN electrode (CNAP), and (c) the macro electrode (macro AP). Note the different scale of the macro AP. The SF recording yields the highest amplitude, whereas the peak-to-peak rise time is about the same (see text).

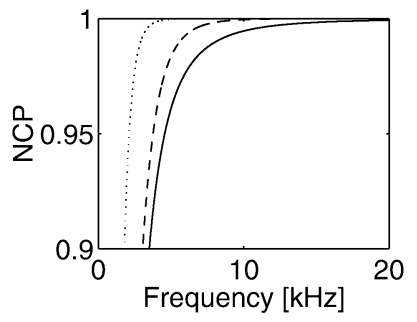

(a)

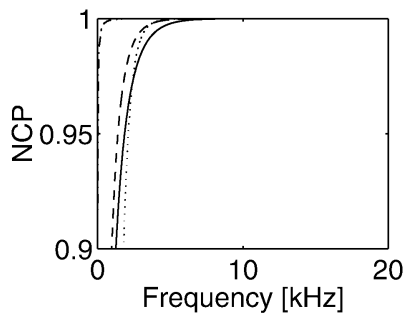

(b)
Fig. 9. (a) Normalized cumulative power (NCP) plot of a transmembrane current both with (dashed) and without (solid) the use of an anti-aliasing Bessel filter. For comparison, the NCP of a SFAP is included (dotted). As can be seen, the unfiltered transmembrane current has substantially more power at higher frequencies compared to the resulting AP. (b) The NCP plot of the weighting functions of a SF electrode (solid), a CN electrode (dashed), and a macro electrode (dash-dotted). For comparison, the NCP of a SFAP is included (dotted). Obviously, all four have their power concentrated to low frequencies.

the $\mathrm{CN}$ electrode (CNAP), and the macro electrode (macro AP), respectively. As expected, the SFAP has the largest amplitude and the most pronounced phases. Moreover, the peak-to-peak rise time is the same for the SFAP and the CNAP. The rise time is slightly larger for the macro AP because the radius of the needle forces a longer distance between the fiber and the electrode.

\section{B. Frequency Distribution}

As stated in Section IV, the large bandwidth of the transmembrane current complicates the choice of discretization frequency because the Nyquist criterion cannot be applied to the AP. Instead, the bandwidth of the transmembrane current and the weighting function introduce constraints on the needed discretization frequency.

As a guide for selecting the correct discretization frequency, the normalized cumulative power (NCP), $\varrho(f)$, is defined as

$$
\varrho(f)=\frac{1}{\int_{0}^{\infty} P(\nu) d \nu} \int_{0}^{f} P(\nu) d \nu
$$

where $P(\nu)$ is the power spectrum of the function under consideration, e.g., $P(\nu)=|I(j 2 \pi \nu)|^{2}$ for the transmembrane current.

In Fig. 9, the NCP is shown for the transmembrane current and the weighting functions. With the NCP of a SFAP included in the figure, the bandwidth difference, as stated in Section IV, of the transmembrane current and the resulting AP is clear. ${ }^{3}$ In Fig. 9(a) we note that, compared to the resulting AP, the transmembrane current has a slower increase in the NCP. This

\footnotetext{
${ }^{3}$ The SFAP is selected for inclusion because it has the highest bandwidth of the three AP types.
} 


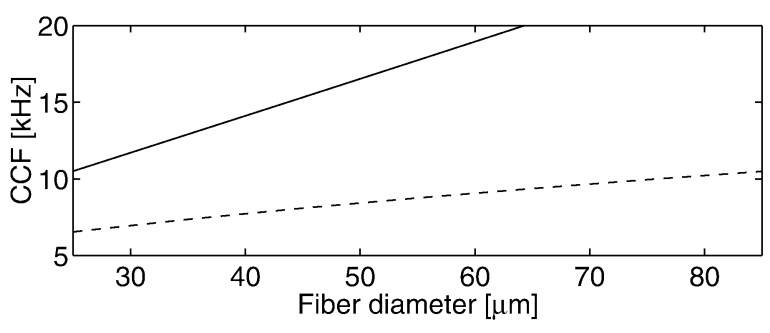

Fig. 10. The CCF, $\varsigma_{0.999}$, below which 0.999 of the total power is found, of the transmembrane current plotted as a function of fiber diameter, and consequently, as a function of conduction velocity. As shown, the CCF is lowered considerably when the anti-aliasing Bessel filter is used (dashed) as compared to the nonfiltered transmembrane current (solid).

means that its spectrum has a slower rolloff, i.e., longer "tails." The bandwidth decrease and the rolloff improvement of the transmembrane current when applying the anti-aliasing filter is significant. The anti-aliasing filter thus decrease the Nyquist frequency, i.e., half of the required discretization frequency, substantially.

Fig. 9(b) shows that both bandwidth and rolloff of the weighting function of the SF electrode and the SFAP are similar. The $\mathrm{CN}$ electrode bandwidth is slightly lower and the macro electrode bandwidth is significantly lower. Therefore, it should be sufficient to low-pass filter the transmembrane current only in order to avoid aliasing while using a discretization frequency equal to the sampling frequency of real APs.

The most important usage of the NCP is to calculate the frequency below which a certain amount of the power is found. We call this frequency the cumulative cutoff frequency $(\mathrm{CCF})$ and it is defined as

$$
\varsigma_{\gamma} \triangleq \nu:[\varrho(\nu)=\gamma], \quad 0 \leq \gamma \leq 1
$$

where $\gamma=0.999$ has shown to provide a reasonable estimate of the required Nyquist frequency.

Using the CCF, it is evident that the Nyquist frequency must not be set according to the bandwidth of the resulting AP. For example, a SFAP with $\varsigma_{0.999}=4.2 \mathrm{kHz}$ may correspond to a transmembrane current with $\varsigma_{0.999}=18 \mathrm{kHz}$. If the Nyquist frequency of the discretization were set by the bandwidth of the resulting AP, considerable aliasing would occur if no anti-aliasing filter was used. This is particularly important when simulating entire MU potentials acquired with a macro electrode because the aliasing errors are "amplified" due to the summed contribution from each fiber [24].

Using the Bessel filter, the $\varsigma_{0.999}=8.8 \mathrm{kHz}$ suggesting that a discretization frequency of $20 \mathrm{kHz}$ is sufficient to avoid aliasing.

From (12), it is apparent that the bandwidth is increased when the conduction velocity increases. Due to the relation between the fiber diameter and conduction velocity (13), the bandwidth depends on the fiber diameter as well. This dependence is shown in Fig. 10, where the CCF, $\varsigma_{0.999}$, of the transmembrane current is plotted as a function of fiber diameter in the range $25-85 \mu \mathrm{m} .{ }^{4}$

All three weighting functions in Fig. 9(b), have their power localized to low frequencies. For the SF electrode $\varsigma_{0.999}=$ $5.9 \mathrm{kHz}$, for the $\mathrm{CN}$ electrode $\varsigma_{0.999}=4.3 \mathrm{kHz}$, and for the

${ }^{4}$ Consequently, conduction velocity ranges from 2.2 to $5.2 \mathrm{~m} / \mathrm{s}$. (a)

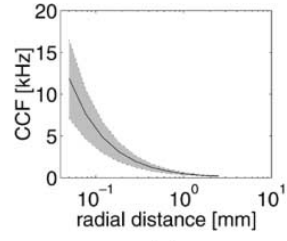

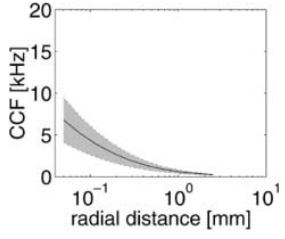

(b)

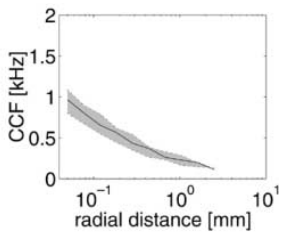

(c)
Fig. 11. The CCF, $\varsigma_{0.999}$, below which 0.999 of the total power of the weighting functions of (a) a SF electrode, (b) a $\mathrm{CN}$ electrode, and (c) a macro electrode is found plotted as a function of radial distance. As NCP frequency depends on the conduction velocity as well, that dependence is shown as a shaded region $(2.2-5.2 \mathrm{~m} / \mathrm{s})$ where the conduction velocity $3.7 \mathrm{~m} / \mathrm{s}$ is shown (solid). Note the different scale of the macro diagram.
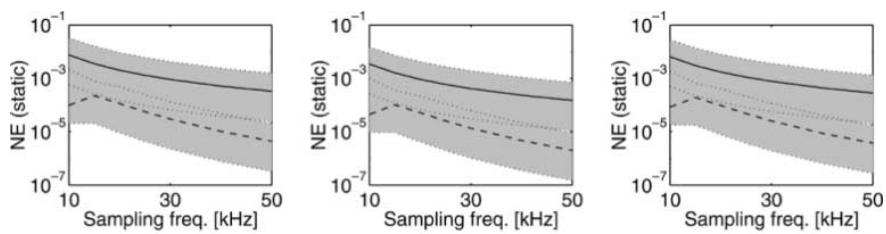

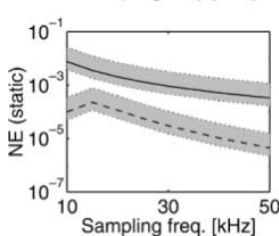

(a)

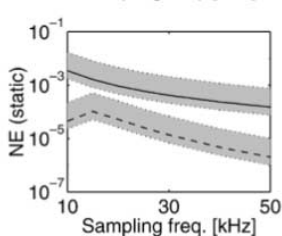

(b)

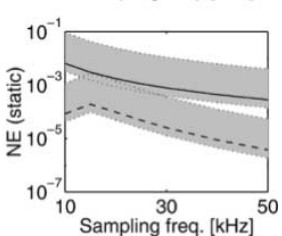

(c)
Fig. 12. Normalized statical error as a function of selected sampling frequency for (a) the SF electrode, (b) the CN electrode, and (c) the macro electrode. The dependence of the fiber diameter (and conduction velocity) is shown (top) as well as the dependence of the radial distance (bottom). The fiber diameter ranges from 25 to $85 \mu \mathrm{m}$. The radial distance ranges from $0.05 \mathrm{~mm}$ to the pick-up distance of each electrode $(0.35,0.5$, and $1.45 \mathrm{~mm}$, respectively). In all figures, the result is shown when no anti-aliasing filter is used (solid) and when a second-order Bessel filter is used (dashed). The $3.7 \mathrm{~m} / \mathrm{s}$ and $0.1 \mathrm{~mm}$ values are plotted in each range. The NE increases if any of $d$ or $r$ increases.

macro electrode $\varsigma_{0.999}=0.74 \mathrm{kHz}$. In Fig. $11, \varsigma_{0.999}$ of the three weighting functions are shown when the radial distance ranges from 0.05 to $2.5 \mathrm{~mm}$. The conduction velocity dependence is visualized with a shaded region where the conduction velocity ranges from 2.2 to $5.2 \mathrm{~m} / \mathrm{s}$. The results in the figure suggest that a discretization frequency of about $20 \mathrm{kHz}$ is sufficient in all cases except when using the SF electrode at small radial distances. In these cases, however, the errors are small compared to the AP and may normally be neglected.

\section{Aliasing Effects}

In Fig. 12, the aliasing effects are shown as a function of discretization frequency. Note that these particular aliasing effects cannot be found in recorded APs because these effects originate from the discretization of the transmembrane current (mainly) and the weighting function. In recorded APs this never occurs because the convolution takes place in continuous time and only the resulting AP is discretized.

The normalized statical error (NE) in Fig. 12 is the dc component due to aliasing 5 multiplied by a normalization factor. The normalization factor is the radial distance divided by a nominal

\footnotetext{
${ }^{5}$ This error is the summed contribution from the spectral components at multiples of the sampling frequency and, hence, constitutes a good measure of the aliasing errors.
} 
distance of $0.1 \mathrm{~mm}$ and accounts for the linear increase of the number of muscle fibers with increasing radial distance.

The figure shows that the statical error is about a factor 100 smaller when the anti-aliasing prefiltering is used compared to when not using it. It is clear from the figure what advantage such a filter has to the discretization frequency requirements.

Moreover, the importance of the aliasing issues when simulating macro APs is clear. The NE is both larger for distant fibers and amplified due to the large number of contributing fibers.

\section{DISCUSSION}

Some novel ideas for simulations using the line source model were presented using a signal processing approach. With this perspective, several improvements to the original model were possible to derive. The two most important of these are the antialiasing filter and the finite-length adjustment.

Regarding the anti-aliasing filter, there has been little attention in the the literature to the aliasing issues when discretizing the model. The referrals made have incorrectly considered the resulting AP in the Nyquist criterion. In this paper, we showed that this may lead to significant aliasing because the bandwidth of the transmebrane current is in general much higher than the bandwidth of the resulting AP.

We presented an approach where an anti-aliasing second order Bessel filter is applied to the transmembrane current analytically. This way the excess bandwidth is decreased and may be used to speed up the simulations, improving the accuracy, or a mix of these. Simulations were used to analyze the aliasing effects and to show the improvements the anti-aliasing filter offers.

Regarding the finite length of the muscle fibers, we presented a simple adjustement to the electrode specific weighting function that improves the efficiency of the simulations by allowing the generated AP being described by a single convolution operation. This is indeed possible with the approach taken in, e.g., [15] but the bandwidth of the corresponding weighting function is then increased. This, in turn, increases the required discretization frequency.

The advantages of using the model as presented in this paper are numerous and here we mention three. First, it is computationally fast and is well suited for simulations of large number of fibers and even entire muscles. Such simulations may contribute to a deeper understanding of the correspondance between the EMG signal and the different neuromuscular disorders. This would improve both research and education as well as clinical work. Second, an anti-aliasing filter is incorporated in the model of the membrane current. With this filter, the discretization frequency may be lowered without affecting the accuracy, and hence, further improving the computational speed. Third, the transfer function representation of the transmembrane current make it possible to use sophisticated signal processing methods on the EMG signal.

The model is already used for simulating MUs as well as entire muscles. A simulation tool [26] has been developed and has shown to be very useful [27], [28].
APPENDIX A

DERIVATION OF THE TEMPORAL WEIGHTING FunCTION

The time-space distribution of the transmembrane current $\xi(t, z)$ is defined in (4). To solve for the end effects $\psi_{i}(t)$, we use that the average transmembrane current along the fiber should be equal to zero [18]. Therefore

$$
\begin{aligned}
0= & \int_{-\infty}^{\infty} \xi(t, z) d z \\
= & \int_{0}^{z_{f}} i_{f}(t, z) d z+\int_{z_{r}}^{0} i_{r}(t, z) d z \\
& +\psi_{0}(t)+\psi_{1}(t)+\psi_{2}(t)
\end{aligned}
$$

To solve (40), two observations are made. First, an end effect is local in its nature. Second, as such, it should be independent of fiber properties at a remote location. The three end effects should thus depend on the end plate position, the first fiber ending, and the second fiber ending, respectively.

By extending (40), the integrals may be written as

$$
\begin{aligned}
0= & \int_{0}^{\infty} i_{f}(t, z) d z-\int_{z_{f}}^{\infty} i_{f}(t, z) d z \\
& +\int_{-\infty}^{0} i_{r}(t, z) d z-\int_{-\infty}^{z_{r}} i_{r}(t, z) d z \\
& +\psi_{0}(t)+\psi_{1}(t)+\psi_{2}(t) .
\end{aligned}
$$

With the observations above, we then conclude that ${ }^{6}$

$$
\begin{aligned}
\psi_{0}(t) & =-\int_{0}^{\infty} i_{f}(t, z) d z-\int_{-\infty}^{0} i_{r}(t, z) d z \\
& =-\int_{0}^{c t} i_{f}(t, z) d z-\int_{-c t}^{0} i_{r}(t, z) d z \\
\psi_{1}(t) & =\int_{z_{f}}^{\infty} i_{f}(t, z) d z=\int_{z_{f}}^{c t} i_{f}(t, z) d z \\
\psi_{2}(t) & =\int_{-\infty}^{z_{r}} i_{r}(t, z) d z=\int_{-c t}^{z_{r}} i_{r}(t, z) d z
\end{aligned}
$$

where we have used that $i_{f}(t, z)=0 \forall z>c t$ and $i_{r}(t, z)=$ $0 \forall z<-c t$.

The final AP is obtained by summing all contributions along the fiber using the weighting function $\bar{w}(z)$ of the measuring electrode

$$
\begin{aligned}
\phi_{c}(t)= & \int_{-\infty}^{\infty} \bar{w}(z) \xi(t, z) d z \\
= & \int_{0}^{z_{f}} \bar{w}(z) i_{f}(t, z) d z+\int_{z_{r}}^{0} \bar{w}(z) i_{r}(t, z) d z \\
& +\bar{w}(0) \psi_{0}(t)+\bar{w}\left(z_{f}\right) \psi_{1}(t)+\bar{w}\left(z_{r}\right) \psi_{2}(t)
\end{aligned}
$$

\footnotetext{
${ }^{6}$ An equivalent result may be obtained by applying Kirchoff's laws to the core conductor model or by taking the approach described in [19].
} 
Inserting $\psi_{i}(t)$ from (42)-(44), $i_{f}(t, z)$, and $i_{r}(t, z)$, we can develop the expression further. Then, we set $z=c \tau$ and obtain

$$
\begin{aligned}
\phi_{c}(t)= & c \int_{0}^{\tau_{f}}(\bar{w}(c \tau)-\bar{w}(0)) i_{c}(t-\tau) d \tau \\
& +c \int_{0}^{\tau_{r}}(\bar{w}(-c \tau)-\bar{w}(0)) i_{c}(t-\tau) d \tau \\
& +c\left(\bar{w}\left(z_{f}\right)-\bar{w}(0)\right) \int_{\tau_{f}}^{t} i_{c}(t-\tau) d \tau \\
& +c\left(\bar{w}\left(z_{r}\right)-\bar{w}(0)\right) \int_{\tau_{r}}^{t} i_{c}(t-\tau) d \tau
\end{aligned}
$$

where

$$
\tau_{f}=\frac{z_{f}}{c} ; \quad \tau_{r}=\frac{-z_{r}}{c}
$$

\section{APPENDIX B}

\section{ANALYTICAL ANTI-ALIASING FILTERING}

Discretizing the source function $i_{c}(t)$, setting $t=n T$, may yield aliasing. By using an anti-aliasing filter, however, this can be reduced. A second-order pole-only filter may be written in the frequency domain on the form

$$
H(s)=\frac{a_{2}}{s^{2}+a_{1} s+a_{2}}
$$

where the coefficients $a_{i}$ determine the filter characteristics.

Applying the filter $H(s)$ in (49) to the source function in (12), we obtain in the frequency domain

$$
\begin{aligned}
\mathcal{L}\left\{i_{c}^{(H)}(t)\right\} & =\mathcal{L}\left\{i_{c}(t)\right\} H(s) \\
& =768 \frac{\sigma_{i} \pi d^{2}}{4} \frac{6 c s^{2}}{(s+2 c)^{4}} \frac{a_{2}}{s^{2}+a_{1} s+a_{2}}
\end{aligned}
$$

Taking the inverse Laplace transform, the filtered source function in the time domain is

$$
\begin{aligned}
i_{c}^{(H)}(t)= & \begin{cases}2304 \frac{\sigma_{i} \pi d^{2}}{4} a_{2} \alpha \varphi(t), & t \geq 0 \\
0, & \text { otherwise } \quad[\mathrm{mV}]\end{cases} \\
\varphi(t)= & C_{0} e^{-\alpha t}+C_{1} t e^{-\alpha t} \\
& +\frac{1}{2} C_{2} t^{2} e^{-\alpha t}+\frac{1}{6} C_{3} t^{3} e^{-\alpha t} \\
& +\left(\frac{\left(\alpha-\frac{1}{2}\right) a_{1} C_{0}-C_{1}}{\beta} \sin (\beta t)\right. \\
& \left.\quad-C_{0} \cos (\beta t)\right) e^{-\left(a_{1} / 2\right) t}
\end{aligned}
$$

where

$$
\begin{aligned}
\alpha & =2 c ; \gamma=\alpha^{2}-a_{1} \alpha+a_{2} \\
C_{0} & =\frac{a_{1} \alpha^{4}-4 a_{2} \alpha^{3}+4 a_{2}^{2} \alpha-a_{1} a_{2}^{2}}{\gamma^{4}} \\
C_{1} & =\frac{a_{1} \alpha^{3}-3 a_{2} \alpha^{2}+a_{2}^{2}}{\gamma^{3}}
\end{aligned}
$$

$$
\begin{aligned}
C_{2} & =\frac{a_{1} \alpha^{2}-2 a_{2} \alpha}{\gamma^{2}} \\
C_{3} & =\frac{\alpha^{2}}{\gamma} \\
\beta & =\sqrt{a_{2}-\frac{1}{4} a_{1}^{2}} .
\end{aligned}
$$

Designing a Bessel filter with cutoff frequency $f_{0}$, the coefficients $a_{i}$ become

$$
\begin{aligned}
\omega & =1.27 ; \quad \zeta=0.87 \\
\omega_{B} & =2 \pi f_{0}\left[\frac{\mathrm{krad}}{\mathrm{s}}\right] \\
a_{1} & =2 \zeta \omega \omega_{B}\left[\frac{\mathrm{krad}}{\mathrm{s}}\right] \\
a_{2} & =\omega^{2} \omega_{B}^{2}\left[\frac{\mathrm{krad}^{2}}{\mathrm{~s}^{2}}\right] .
\end{aligned}
$$

\section{ACKNOWLEDGMENT}

The authors would like to thank Prof. A. Ahlén and Prof. M. Sternad, both with Signals and Systems, for valuable comments regarding this work.

\section{REFERENCES}

[1] A. D. Adrian and D. W. Bronk, "The discharge of impulses in motor nerve fibers. The frequency of discharges in reflex and voluntary contractions," J. Physiol. Lond., vol. 67, pp. 119-151, 1929.

[2] E. Stålberg, S. D. Nandedkar, D. B. Sanders, and B. Falck, "Quantitative motor unit potential analysis," J. Clin. Neurophysiol., vol. 13, pp. 401-422, 1996

[3] E. Stålberg and J. V. Trontelj, Single Fiber Electromyography in Healthy and Diseased Muscle, 2nd ed. New York: Raven, 1994.

[4] J. Ekstedt, P. Häggqvist, and E. Stålberg, "The construction of needle multi-electrodes for single fiber electromyography," Electroencephalogr. Clin. Neurophysiol., vol. 27, pp. 540-543, 1969.

[5] E. Stålberg, "Macro EMG, a new recording technique," J. Neurol. Neurosurg. Psych., vol. 43, pp. 475-482, 1980.

[6] R. Lorente de Nó, "A study of nerve physiology," in Analysis of the Distribution of Action Currents of Nerve in Volume Conductors: Studies from the Rockefeller Institute for Medical Research, 1947, vol. 132, ch. XVI, pp. 384-477.

[7] J. Clark and R. Plonsey, "The extracellular potential field of the single active nerve fiber in a volume conductor," Biophys. J., vol. 8, pp. 842-864, 1968

[8] P. Rosenfalck, "Intra and extracellular potential fields of active nerve and muscle fibers. A physio-mathematical analysis of different models," Acta Physiol. Scand., vol. 321, pp. 1-168, 1969.

[9] P. A. M. Griep, K. L. Boon, and D. F. Stegeman, "A study of the motor unit action potential by means of computer simulation," Biol. Cybern., vol. 30, pp. 221-230, 1978.

[10] J. Ekstedt and E. Stålberg, "How the size of the needle electrode leading-off surface influences the shape of the single muscle fiber action potential in electromyography," Comp. Prog. Biomed., vol. 3, pp. 204-212, 1973.

[11] D. C. Boyd, P. D. Lawrence, and P. J. A. Bratty, "On modeling the single motor unit action potential," IEEE Trans. Biomed. Eng., vol. BME-25, pp. 236-243, 1978.

[12] S. D. Nandedkar and E. V. Stålberg, "Simulation of single muscle fiber action potentials," Med. Biol. Eng. Comput., vol. 21, pp. 158-165, Mar. 1983.

[13] S. Andreassen and A. Rosenfalck, "Relationship of intracellular and extracellular action potential of skeletal muscle fiber," CRC Crit. Rev. Bioeng., vol. 6, pp. 267-306, Nov. 1981.

[14] T. H. Gootzen, "Muscle fiber and motor unit action potentials: A biophysical basis for clinical electromyography," Ph.D. dissertation, Univ. Nijmegen, Nijmegen, The Netherlands, 1990. 
[15] G. V. Dimitrov and N. A. Dimitrova, "Precise and fast calculation of the motor unit potentials detected by a point and a rectangular plate electrode," Med. Eng. Phys., vol. 20, pp. 374-381, 1998.

[16] K. C. McGill, Z. C. Lateva, and S. Xiao, "A model of the muscle action potential for describing the leading edge, terminal wave, and slow afterwave," IEEE Trans. Biomed. Eng., vol. 48, pp. 1357-1365, Dec. 2001.

[17] A. Gydikov and N. Trayanova, "Extracellular potentials of single active muscle fibers: Effects of finite fiber length," Biol. Cybern., vol. 53, pp. 363-372, 1986.

[18] T. H. Gootzen, D. F. Stegeman, and A. van Oosterom, "Finite limb dimensions and finite muscle length in a model for the generation of electromyographic signals," Electroencephalogr. Clin. Neurophysiol., vol. 81, pp. 152-162, Apr. 1991.

[19] J. Duchêne and J.-Y. Hogrel, "A model of EMG generation," IEEE Trans. Biomed. Eng., vol. 47, pp. 192-201, Feb. 2000.

[20] B. K. van Veen, H. Wolters, W. Wallinga, W. L. C. Rutten, and H. B. K. Boom, "The bioelectrical source in computing single muscle fiber action potentials," Biophys. J., vol. 64, pp. 1492-1498, 1993.

[21] S. D. Nandedkar, E. V. Stålberg, and D. B. Sanders, "Simulation techniques in electromyography," IEEE Trans. Biomed. Eng., vol. BME-32, pp. 775-785, Oct. 1985.

[22] R. Plonsey, "Dependence of scalar potential measurements on electrode geometry," Rev. Sci. Instrum., vol. 36, 1965.

[23] M. M. H. J. Theeuwen, T. H. J. M. Gootzen, and D. F. Stegeman, "Muscle electric activity I: A model study on the effect of needle electrodes on single fiber action potentials," Ann. Biomed. Eng., vol. 21, no. 4, pp. 377-389, 1993.

[24] S. D. Nandedkar and E. V. Stålberg, "Simulation of macro EMG motor unit potentials," Electroencephalogr. Clin. Neurophysiol., vol. 56, pp. 52-62, Feb. 1983

[25] S. D. Nandedkar, D. B. Sanders, E. V. Stålberg, and S. Andreassen, "Simulation of concentric needle EMG motor unit action potentials," Muscle Nerve, vol. 11, pp. 151-159, Feb. 1988

[26] L. Karlsson, B. Hammarberg, and E. Stålberg, "An application of a muscle model to study electromyographic signals," Comp. Meth. Prog. Biomed., vol. 71, no. 3, pp. 225-233, July 2003.

[27] E. Stålberg and L. Karlsson, "Simulation of the normal concentric needle electromyogram by using a muscle model," Clin. Neurophysiol., vol. 112, pp. 464-471, Mar. 2001.

[28] — "Simulation of EMG in pathological situations," Clin. Neurophysiol., vol. 112, pp. 869-878, May 2001.

[29] P. A. M. Griep et al., "Calculation and registration of the same motor unit action potential," Electroencephalogr. Clin. Neurophysiol., vol. 53, pp. 388-404, 1982.
[30] D. Dumitru, "Electrophysiologic basis for single muscle fiber discharge morphology," Ph.D. dissertation, Univ. Nijmegen, Nijmegen, The Netherlands, 1999.

[31] T. H. Gootzen, D. F. Stegeman, and A. van Oosterom, "Finite limb dimensions and finite muscle length in a model for the generation of electromyographic signals," Ann. Biomed. Eng., vol. 21, pp. 391-399, July 1993.

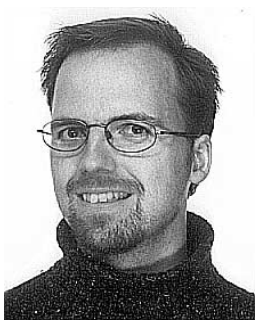

Björn Hammarberg (Hansson) (S'00-M'02) was born in Östersund, County of Jämtland, Sweden, in 1970. He received the M.Sc. degree in engineering physics and the Ph.D. degree from Uppsala University, Uppsala, Sweden, in 1995 and in 2002, respectively. His Ph.D. studies were in signal processing of neurophysiological data.

From 1995 through 2002, he was with the Department of Clinical Neurophysiology at University Hospital, Uppsala, working with signal processing in various medical applications. During the fall of 1995, he was with the Department of Clinical Neurophysiology, Institute of Neurology, Nijmegen University Hospital, the Netherlands, working on an EMG decomposition system.

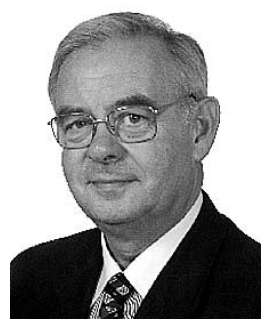

Erik Stålberg was born in Skellefteå, Sweden, in 1936. He received the Ph.D. degree from Uppsala University, Uppsala, Sweden, in 1966.

In 1991, he became Professor and Chairman of the Department of Clinical Neurophysiology, University Hospital Uppsala, where, since 2001, he has held the position of Professor Emeritus. His main research interest has been the study of the human motor unit in health and disease. He has developed EMG methods such as single fiber EMG, macro EMG, and scanning EMG. Another area of his interest has been the development of quantitative methods for the analysis of the motor unit potentials and he is, therefore, also active in the field of signal analysis. 